\title{
Particularidades do diagnóstico e da terapêutica da síndrome dos ovários policísticos na adolescência
}

\author{
Particularities of the diagnosis and therapy of polycystic ovary syndrome in adolescence
Particularidades del diagnóstico y terapia del síndrome de ovario poliquístico en la adolescencia

Giovanna Rocha Lisboa ${ }^{*}$, Laís Kemelly Lima de Araújo², Lais Marinho Rosa ${ }^{3}$, Laís Vieira Brito4, Larissa Diogo Viana Maciel ${ }^{5}$, Layane Aiala de Sousa Lopes ${ }^{6}$, Lorena Mendonça Silveira ${ }^{7}$, Otávio Ramalho ${ }^{4}$, Rebeca Oliveira Rocha ${ }^{8}$, Anna Carla Garcia Cabral ${ }^{9}$.

\section{RESUMO}

Objetivo: Avaliar o processo diagnóstico e terapêutico, incluindo as manifestações clínicas e fisiológicas em adolescentes que sofrem com a Síndrome do Ovário Policístico (SOP). Revisão bibliográfica: A SOP, caracteriza por um distúrbio endocrinológico que afeta mulheres na idade fértil. No entanto, divergências nos estudos sobre a causa da SOP, promove a formação de hipóteses sobre o que provocaria o desequilíbrio hormonal, tendo a origem genética como possível explicação. Além disso, a dificuldade da realização do diagnóstico precocemente, torna-se mais um fator agravante na eficácia do tratamento. Desse modo, as manifestações clínicas, reprodutivas e metabólicas interferem na qualidade de vida em curto prazo com irregularidade do sangramento uterino e cólicas recorrentes, além de complicações em longo prazo como o câncer endometrial. Considerações finais: Conclui-se ser importante o diagnóstico da síndrome dos ovários policísticos, visto que se trata de uma síndrome que está relacionada a diversas comorbidades, podendo gerar repercussões metabólicas e reprodutivas nessas mulheres. Portanto, o tratamento deve ser individualizado e de caráter multifatorial, considerando as queixas da paciente, o estágio da vida reprodutiva em que se encontra e avaliando o risco/benefício de determinadas terapias.

Palavras-chave: Síndrome do ovário policístico, Adolescente, Diagnóstico.

\section{ABSTRACT}

Objective: To evaluate the diagnostic and therapeutic process, including the clinical and physiological manifestations in adolescents suffering from Polycystic Ovarian Syndrome (PCOS). Bibliographic review: SOP is characterized by an endocrinological disorder that affects women of childbearing age. However, divergences in studies on the cause of SOP, which promotes the formation of hypotheses on what would cause the hormonal imbalance, having the genetic origin as a possible explanation. In addition, the difficulty of making the diagnosis early becomes another aggravating factor in the effectiveness of treatment. Thus, the clinical, reproductive and metabolic manifestations interfere in the quality of life in the short term with irregularity of uterine bleeding and recurrent colic, in addition to long-term complications such as endometrial cancer. Final considerations: It is concluded that the diagnosis of the polycystic ovary syndrome is important, since it is a syndrome that is related to several comorbidities and may generate metabolic and reproductive repercussions in these women. Therefore, the treatment must be individualized and of multifactorial character, considering the complaints of the patient, the stage of reproductive life in which she is and evaluating the risk/benefit of certain therapies.

Keywords: Polycystic ovary syndrome, Adolescent, Diagnosis.

\footnotetext{
1 Universidade de Gurupi, Gurupi - TO. *E-mail: giovanna3008@gmail.com

2 União Metropolitana para Educação e Cultura (UNIME), Lauro de Freitas - BA.

${ }^{3}$ Universidade de Franca (UNIFRAN), Franca - SP.

${ }^{4}$ Faculdade de Minas (FAMINAS-BH), Belo Horizonte - MG.

${ }^{5}$ Faculdade de Medicina de Valença (FMV), Valença - RJ.

${ }^{6}$ Faculdade Metropolitana São Carlos (FAMESC), Bom Jesus do Itabapoana - RJ.

${ }^{7}$ Centro Universitário do Planalto Central Apparecido dos Santos (UNICEPLAC), Brasília - DF.

8 Universidad Cristiana de Bolivia (UCEBOL), Santa Cruz de La Sierra, Bolívia

9 Universidade Católica de Brasília, Brasília - DF.
} 


\section{RESUMEN}

Objetivo: Evaluar el proceso diagnóstico y terapéutico, incluyendo las manifestaciones clínicas y fisiológicas en adolescentes que padecen el Síndrome de Ovarios Poliquísticos (SOP). Revisión bibliográfica: EI SOP se caracteriza por ser un trastorno endocrinológico que afecta a las mujeres en edad fértil. Sin embargo, las diferencias en los estudios sobre la causa del SOP promueven la formación de hipótesis sobre lo que causaría el desequilibrio hormonal, con el origen genético como posible explicación. Además, la dificultad de realizar el diagnóstico precozmente se convierte en otro factor agravante de la eficacia del tratamiento. Así, las manifestaciones clínicas, reproductivas y metabólicas interfieren en la calidad de vida a corto plazo con la irregularidad de la hemorragia uterina y los cólicos recurrentes, además de las complicaciones a largo plazo como el cáncer de endometrio. Consideraciones finales: Se concluye que el diagnóstico del síndrome de ovario poliquístico es importante, ya que es un síndrome que se relaciona con varias comorbilidades y puede generar repercusiones metabólicas y reproductivas en estas mujeres. Por lo tanto, el tratamiento debe ser individualizado y de carácter multifactorial, considerando las dolencias de la paciente, la etapa de la vida reproductiva en la que se encuentra y evaluando el riesgo/beneficio de determinadas terapias.

Palabras clave: Síndrome del ovario poliquístico, Adolescente, Diagnóstico.

\section{INTRODUÇÃO}

A Síndrome do Ovário Policístico (SOP), se caracteriza por um distúrbio endocrinológico que afeta mulheres na idade fértil, apresentando sua prevalência entre 6 a 16\% dependendo da população estudada e dos critérios diagnósticos utilizados. Além disso, é comumente associada a enfermidades importantes que influenciam no bem-estar físico, psicológico e financeiro em longo prazo (DOKRAS A, et al., 2016; ROSA-ESILVA AC, 2018).

De acordo com Gowri BV, et al. (2015), a SOP trata-se de uma enfermidade reconhecida por suas manifestações clínicas cutâneas, reprodutivas e metabólicas. Existe uma alteração hormonal em decorrência do excesso de produção de andrógenos desencadeando uma sintomatologia como oligo ou anovulação, hirsutismo, acne, alopecia androgenética, seborréia.

A manifestação dessa enfermidade durante a adolescência é mais frequente do que se espera, com base em estudos de Yela DA (2018) e Rosa-e-Silva AC (2018), estima-se que, a incidência de SOP ao avaliar os parâmetros como alteração menstrual, aumento de androgênios e alteração morfológica em ultrassonografia em uma população de 244 adolescentes é de 18,5\% de acordo aos critérios do consenso de Rotterdan, onde se é necessário 2 de 3 parâmetros e nenhum é obrigatório. Com respeito aos critérios da Sociedade de Excesso Androgênico (AES-PCOS), a prevalência é de 5\%, uma vez que somente o hiperandrogenismo é considerado um critério obrigatório. Quando se analisa a população com os critérios do National Institute Health $(\mathrm{NHI})$, onde o hiperandrogenismo e aligo-amenorreia são critérios obrigatórios, se obteve a incidência de 3,1\% de adolescentes (FREIRE A, et al., 2018; YELA DA, 2018).

A manifestação dessa enfermidade durante a adolescência é mais frequente do que se espera. $O$ estudo de Yela DA (2018) afirma que uma população de 244 adolescentes caucasianas entre 14 e 16 anos, há uma prevalência de SOP de 18,5\% segundo critérios do Consenso de Rotterdam, de $5 \%$ de acordo com os critérios da Sociedade de Excesso Androgênio e de 3,1\% segundo os do National Institute Health (NHI), (YELA DA, 2018). No entanto, o estudo afirma ainda que existem alguns fatores que contribuem para o desenvolvimento da SOP durante essa fase, como o aumento do fator de crescimento semelhante à insulina I (IGF-1) e da insulina, além de apresentar relações com o baixo peso ao nascer e pubarca precoce (FREIRE A, et al., 2018; YELA DA, 2018).

Porém, existe uma dificuldade no diagnóstico rápido e adequado da SOP durante a adolescência devido as mudanças fisiológicas comumente encontradas nessa faixa etária, faz com que os sintomas e sinais da síndrome sobreponham tais alterações (FREIRE A, et al., 2018; YELA DA, 2018).

Por se tratar de uma síndrome com afetação metabólica, reprodutiva e relacionada a outras comorbidades, como obesidade, diabetes tipo 2, infertilidade, hiperplasia endometrial, hipertensão arterial, entre outros, é de suma importância o diagnóstico correto. Com isso, o consenso de Rotterdam, em 2003, estabelece a 
necessidade da presença dos três critérios: 1) alteração menstrual, 2) hiperandrogenismo e 3) presença da morfologia de ovário policístico em ultrassonografia a fim de determinar um diagnóstico durante a adolescência (FREIRE A, et al., 2018; YELA DA, 2018; WINNYKAMIEN I, et al., 2017).

Dessa forma, assimilando as manifestações clínicas da síndrome e suas implicações durante essa etapa da vida, é necessário proporcionar uma terapêutica correspondente e individualizada de acordo com as afetações de cada paciente, levando em consideração suas necessidades, visando sempre melhorar a qualidade de vida e prevenir comorbidades (FREIRE A, et al., 2018).

Logo, o tratamento, segundo Yela DA (2018), consiste primeiramente em uma abordagem não farmacológica, que resulta em dieta hipocalórica visando a perda de peso e redução de medidas da circunferência abdominal. Uma vez que, é comprovada a relação entre a perda de peso e regulação do sistema metabólico e endócrino, onde se diminui a resistência insulínica, dislipidemia, e o nível de testosterona, e aumenta a concentração de globulina ligadora dos hormônios sexuais (SHBG).

As medidas farmacológicas se baseiam no uso de anticoncepcionais orais combinados, que atuam tanto nas manifestações androgênicas como também na alteração menstrual, ou apenas progestagênios isolados de maneira cíclica, cujo sua eficácia é somente para a irregularidade menstrual. Esses anticoncepcionais podem estar associados ou não ao uso de metformina com a finalidade de melhorar os parâmetros metabólicos apresentados pela SOP (FREIRE A, et al., 2018; YELA DA, 2018).

Diante do exposto, o objetivo do presente estudo é evidenciar as principais manifestações clínicas da síndrome do ovário policístico e identificar as particularidades do diagnóstico e da terapêutica durante a adolescência, por meio de uma revisão bibliográfica detalhada do tema, analisando e explicando as principais características da problemática na saúde feminina.

\section{REVISÃO BIBLIOGRÁFICA}

\section{Fisiopatologia}

A fisiopatologia da SOP consiste inicialmente em alterações no eixo hipotálamo-hipófise, na pulsatilidade do hormônio liberador de gonadotrofina $(\mathrm{GnRH})$, secretado pelo hipotálamo que estimula a adenohipófise a secretar Hormônio Luteinizante (LH) e Hormônio Folículo Estimulante (FSH), como lbáñeza L, et al. (2017) descreve, são hormônios que controlam a esteroidogênese ovariana, dinâmica folicular e ovulação.

De acordo com os estudos realizados por Batista LB, et al., (2019), ficou comprovado que uma característica consistente das pacientes com SOP é um quadro de secreção desordenada de gonadotrofina com LH médio elevado, FSH normal/baixo ou baixo e uma taxa persistentemente rápida de secreção de pulso de GnRH (BATISTA LB, et al., 2019).

Portanto, uma vez que o LH desempenha função de estimular as células da teca a produzirem andrógenos, e o FSH estimula as células da granulosa a fazer a conversão de androgênios para estrogênios pela ação da enzima aromatase, as alterações neuroendócrinas descritas acima justificam o porquê do excesso de andrógenos, observado em aproximadamente $60-80 \%$ das pacientes com SOP, que é uma característica fundamental do distúrbio (IBÁÑEZA L, et al., 2017).

O desenvolvimento folicular normal é essencial para que ocorra o processo de ovulação, pois durante seu estágio de maturação ele se transformará em um folículo pré-ovulatório, por influências do LH e FSH. Diante disso, se o ovário tiver influências androgênicas, ocorrerá atresia folicular e consequentemente anovulação, característico da SOP, pela falta de folículos maduros. Se não há ovulação não há corpo lúteo, consequentemente não há produção de progesterona, o endométrio dessa paciente será estimulado somente pelo estrogênio, levando ao risco de sangramento uterino anormal, hiperplasia endometrial, câncer de endométrio (IBÁÑEZA L, et al., 2017; BATISTA LB, et al., 2019).

A partir da produção científica que foi base para a presente investigação, observou-se que na SOP o equilíbrio entre os andrógenos, o Hormônio anti-Mülleriano (AMH) e o FSH é interrompido levando a parada folicular. As concentrações de FSH e a conversão de andrógenos em estradiol são insuficientes, resultando 
em falha na seleção de um folículo maduro, portanto, anovulação crônica. $\mathrm{OAMH}$, secretado pelas células da granulosa, desempenha um papel importante no controle desse equilíbrio, pois inibe a transição dos folículos primordiais para os primários. Desta forma, a SOP é caracterizada por aumento do crescimento de pequenos folículos, mas subsequente parada do crescimento levando à morfologia policística típica (IBÁÑEZA L, et al., 2017).

Existe também uma maior probabilidade de pacientes com SOP apresentarem resistência insulínica, afetando $40-75 \%$ das mulheres. Isso se dá porque além de se tratar de um hormônio metabólico, a insulina também é considerada um hormônio reprodutivo, pela sua atuação direta em receptores insulínicos que se encontra nos tecidos ovarianos. Portanto, atua diretamente na liberação anormal de LH e FSH, potencializando o efeito da esteroidogênese. Além da sua contribuição na diminuição da síntese hepática de proteínas transportadoras de fatores de crescimento insulínico 1 (IGFBP-1) e da globulina ligadora dos hormônios sexuais (SHGB), o que induz o aumento da produção de andrógenos livres (FUNG L, et al., 2015; PEÑA GM, et al., 2019).

Rodriguez JH e Puig MEL (2015), também associa a presença da SOP em mulheres com maior quantidade de grasa visceral, devido a liberação de adipocinas proinflamatórias, como interleucina-6 (IL-6), fator de necrose tumoral a (TNF-a), e também ocorre uma diminuição de adipocinas anti-inflamatórias, como a adiponectina, desencadeando a patogênese da resistência à insulina (RODRÍGUEZ JH e PUIG MEL, 2015).

Sendo a insulino-resistência um fator agravante na patogenia da SOP, estudos demonstram melhor prognóstico em pacientes que fizeram uso de insulino sensibilizadores como a metformina, associado às mudanças de hábitos de vida. Pois a redução de peso também é fator que contribui no decaimento dos sintomas, além de atuar diretamente na prevenção das comorbidades cardiovasculares e diabetes mellitus tipo II (RODRÍGUEZ JH e PUIG MEL, 2015).

Existem outras manifestações causadas pelo hiperandrogenismo (acne, hirsutismo e/ou alopecia androgênica), comumente demonstrado por níveis elevados de testosterona livre em circulação é a anormalidade mais comum observada na síndrome e desempenha um papel importante na perpetuação dos contribuintes hormonais aberrantes para a fisiopatologia da SOP. Um exemplo disso é o hirsutismo, o que faz engrossar e enegrecer os pelos de áreas sensíveis aos andrógenos é a testosterona livre dentro do folículo piloso (IBÁÑEZA L, et al., 2017).

\section{Manifestações Clínicas}

Tendo em vista a metanálise realizada por Bozdag G, et al., (2016), concluiu-se que dentre as possíveis manifestações clínicas no cenário heterogêneo da SOP, as mais frequentes seriam: a oligoanovulação, o hiperandrogenismo clínico, a presença de morfologia de ovário policístico ao ultrassom (MOP) e o hiperandrogenismo bioquímico. O diagnóstico de SOP torna-se mais desafiador na população adolescente, haja vista que a irregularidade menstrual e ovários multifoliculares fazem parte da fisiologia puberal (TAY CT, et al., 2020).

$\mathrm{Na}$ visão de Freire A, et al. (2020), o hiperandrogenismo clínico pode ser avaliado a partir de sua manifestação pilossebácea, ou seja, a presença de acne moderada/severa irresponsiva a tratamentos prévios. Já, conforme Villacis SC, et al. (2017), o hirsutismo também é um indicativo de hiperandrogenismo e seu diagnóstico é realizado a partir da escala de Ferriman-Galley, a partir da qual a adolescente é avaliada nas seguintes regiões: lábio superior, mento, peito, costas superior e inferior, abdômen superior e inferior, braço, antebraço, coxa e perna, recebendo um ponto para cada região em que a distribuição dos pelos é tipicamente masculina, sendo que uma pontuação a partir de 8 é considerada indicativo de hirsutismo.

A partir dos dados apresentados por Villacis SC, et al. (2017), a oligomenorreia deve ser cautelosamente avaliada, a fim de distinguir a anovulação fisiológica dos dois primeiros anos após a menarca, da verdadeira anovulação. Nesse sentido, Freire A, et al. (2018) leva em consideração os seguintes critérios: a. Intervalo > 90 dias no $1^{\circ}$ ano pós-menarca; b. Intervalo $>45$ dias no $2^{\circ}$ ano pós-menarca; c. Ausência de menarca aos 15 anos com 2-3 anos de intervalo após a telarca. 
Portanto, os estudos demonstraram que em relação à MOP, a mesma é diagnosticada quando há presença de 12 ou mais folículos medindo entre $2-9 \mathrm{~mm}$ e/ou volume ovárico $>10 \mathrm{~mm}^{3}$. No entanto, o padrão multifolicular pode estar presente de forma fisiológica nas adolescentes como consequência de sua adaptação fisiológica puberal (VILLACIS SC, et al., 2017). Segundo Freire A, et al. (2018), o hiperandrogenismo laboratorial pode ser diagnosticado a partir da dosagem sérica da testosterona total e de sua fração livre, sendo considerados achados de hiperandrogenismo os níveis de $>50 \mathrm{ng} / \mathrm{dL}$ e $>24 \mathrm{pmol} / \mathrm{L}$, respectivamente.

\section{Os três critérios e as possíveis complicações}

De acordo com Tay CT, et al. (2020), três critérios diagnósticos já foram estabelecidos para diagnosticar a Síndrome dos Ovários Policísticos (SOP), levando em consideração suas manifestações clínicas mais comuns. Em 1990, a National Institutes of Health (NIH) considerava que a presença de hiperandrogenismo clínico ou laboratorial associada à oligoamenorreia.

Segundo Freire A, et al. (2018), o diagnóstico de SOP, além de ser um diagnóstico de exclusão, se baseia nos Critérios de Rotterdam, os quais foram elaborados em 2003 e incluem, o hiperandrogenismo, a irregularidade menstrual e a morfologia do ovário policístico ao exame de ultrassom, sendo necessário para a confirmação diagnóstica a presença de pelo menos 2 destes critérios.

$\mathrm{Na}$ fase de adolescência, porém, meninas saudáveis podem apresentar a clínica desses critérios e isso ser apenas um quadro típico de alterações fisiológicas confundindo o diagnóstico. Tendo em vista essa variável, o Guideline Internacional de SOP recomenda que a morfologia de ovários policísticos seja evitada para fins diagnósticos, preconizando a apresentação clínica de hiperandrogenismo e oligomenorreia (TAY CT, et al., 2020).

Para facilitar o diagnóstico na adolescência os elementos clínicos incluem o hirsutismo distribuídos em áreas masculinas, a presença de acne de caráter inflamatório moderado ou grave e a resistência ao tratamento tópico da mesma. A irregularidade menstrual não característica dessa idade ginecológica é baseada em ciclos menstruais consecutivos $>90$ dias no primeiro ano após a primeira menstruação, ou então períodos contínuos > 45 dias em 2 anos no ou mais depois da primeira menstruação ou, por fim, a ausência de ciclos aos 15 anos já tendo desenvolvido telarca (FREIRE A, et al., 2018).

Em relação ao papel do ultrassom pélvico no diagnóstico diferencial da SOP na adolescência, o mesmo não se encaixa, visto que é comum o aparecimento de ovários policísticos nessa idade pós-menarca, assim é utilizado apenas para descartar outras patologias. Para auxiliar na confirmação de hiperandrogenemia, exames laboratoriais como dosagem de testosterona total (>50 ng/dL) ou testosterona livre (>24 pmol/ L) podem ser úteis (FREIRE A, et al., 2018).

Segundo Winnykamien I, et al. (2017), as mulheres com SOP possuem comorbidades como obesidade, dislipidemia, intolerância à glicose, diabetes mellitus tipo 2, hipertensão arterial e transtorno de humor, sendo necessário a avaliação das mesmas a fim de evitar a ocorrência de doenças cardiovasculares. Em concordância a isso, Batista LB, et al. (2019) evidenciou a associação de calcificação das artérias coronárias e espessura da íntima média da carótida a ocorrências de eventos cardíacos.

Além disso, ressalta-se possíveis consequências clínicas que são secundárias a anovulação. Uma delas pode ser a hiperplasia ou câncer de endométrio, caracterizada pelo estímulo contínuo de estrogênio ao endométrio na ausência de progesterona, induzindo assim sua proliferação, sendo o ideal realizar uma avaliação endometrial após o diagnóstico de SOP. A osteoporose, uma segunda consequência que ainda não foi comprovada, mas que já foi relatada em alguns estudos a sua relação com quadros crônicos. A esterilidade, muitas vezes o marco para busca do médico, trata-se de uma consequência em curto prazo de tal patologia (LESTER LB, et al., 2019).

Kogure GS e Reis RM (2017), afirmam que na vida adulta, a patologia está associada com desfechos que vão além dos impactos reprodutivos, como: disfunções psicossociais, estresse, depressão e ansiedade.

\section{Influência da SOP na adolescência}

As transformações fisiológicas que ocorrem na puberdade provocam marcas em curto e longo prazo. Os reflexos dessas transformações são perceptíveis, com ênfase no componente emocional, uma vez que a 
capacidade reprodutiva feminina é, marcadamente, prejudicada. Sob esse aspecto, nota-se que para as adolescentes que possuem SOP, além das preocupações e agonias típicas dessa etapa do desenvolvimento, ainda precisam estabelecer uma maturação psicossocial precoce para lidar com uma doença que altera o estilo de vida, gerando ansiedades e frustrações (BLAY AL, et al., 2016). O impacto emocional influencia, negativamente, na construção da autoimagem e da autoestima, propiciando a ocorrência de distúrbios psíquicos, como bulimia, anorexia nervosa e depressão. As transformações físicas (ganho de peso e acne) distorcem a imagem corporal das adolescentes, de modo a interferir na sexualidade, repercutindo em insegurança e danos psicológicos que refletirão na vida adulta (BLAY AL, et al., 2016).

Portanto percebe-se a necessidade de um atendimento multifocal, colocando as necessidades emocionais niveladas as fisiológicas, além de correlacionar o conhecimento de saúde sexual, principalmente, porque muitos pacientes não possuem vida sexual ativa. Nesse sentido, é bom ressaltar a importância do cuidado emocional estar nivelado ao cuidado fisiológico, ou seja, proporcionar assistência e informações, a fim de amenizar os sintomas, garantir adesão ao tratamento e estimular um sucesso terapêutico durante o transcorrer da adolescência para a vida adulta (BLAY SL, et al., 2016).

\section{As opções de tratamento disponíveis}

O manejo das pacientes com SOP deve ser de caráter multifatorial com ênfase no tratamento dos fatores clínicos prevalentes na manifestação da doença, levando em consideração o estágio de vida reprodutiva em que as mulheres se encontram. Nesse contexto, as decisões de tratamento da SOP são tomadas de acordo com as prioridades das pacientes, com as possíveis eficácias e riscos que algumas terapias podem oferecer (BATISTA LB, et al., 2019). Portanto, os alvos terapêuticos se relacionam principalmente em evitar infertilidade, controlar o ciclo menstrual e os quadros de hirsutismo, assim como as alterações metabólicas (McCARTNEY CR e MARSHALL JC, 2016).

Nenhum tratamento farmacológico possui recomendações e a devida aprovação pela Food and Drug Administration (FDA) para uso em adolescentes com SOP, entretanto, algumas intervenções farmacológicas têm sido usadas para gerenciar os sintomas da doença (IBÁÑEZA L, et al., 2017).

A associação da prática intensificada de exercícios físicos com a perda de peso foi benéfica na melhora do quadro da SOP de adolescentes com a doença na presença de sobrepeso sem promover efeitos colaterais (HOEGER K, et al., 2008; ORNSTEIN RM, et al., 2011; LASS N, et al., 2011).

O tratamento de distúrbios relacionados à ovulação e a infertilidade em pacientes com SOP inclui diversas opções, como o uso de moduladores do receptor de estrogênio (clomifeno e tamoxifeno), inibidores da aromatase (letrozol), estimulação hormonal direta dos ovários (gonadotrofinas) e medicamentos sensibilizadores de insulina (metformina) (IBÁÑEZA L, et al., 2017; WITCHEL SF, et al., 2015).

Além disso, pacientes com SOP e excesso de peso devem ser conscientizadas para que possam ter abordagens que resultem na perda de peso, considerando reduzir a resistência à níveis de insulina e testosterona livres e, consequentemente, melhorando a regularidade menstrual e as taxas de ovulação e gravidez (BATISTA LB, et al., 2019; IBÁÑEZA L, et al., 2017).

A terapêutica do hiperandrogenismo é uma das etapas de extrema relevância na terapêutica da SOP por meio do controle do hirsutismo. De forma geral, ela se baseia em protocolos terapêuticos que têm como finalidade principalmente a diminuição da produção de androgênios endógenos e em procedimentos de caráter não hormonais, ou seja, cosméticos-depilatórios (BATISTA LB, et al., 2019).

Dessa maneira, medidas cosméticas depilatórias como, remoção dos cabelos por eletrólise, termólise e uso de laser costumam ser eficazes de maneira individualizada no controle do hirsutismo de grau leve e localizado, enquanto nos casos clínicos moderados e graves geralmente são associadas a tratamentos farmacológicos que envolvem o uso anticoncepcionais orais combinados, produtos que contenham progestágenos com baixo potencial androgênico/efeitos antiandrogênicos, compostos de estrogênioprogestina, entre outros (BATISTA LB, et al., 2019; IBÁÑEZA L, et al., 2017). 


\section{CONSIDERAÇÕES FINAIS}

O presente trabalho evidenciou a importância do diagnóstico da síndrome dos ovários policísticos, visto que se trata de uma síndrome que está relacionada a diversas comorbidades, como obesidade, diabetes, hipertensão, hiperplasia endometrial e infertilidade, podendo gerar repercussões metabólicas e reprodutivas nessas mulheres. Além disso, há dificuldade para o diagnóstico rápido e adequado da síndrome, pois adolescentes saudáveis podem apresentar alterações fisiológicas que fazem parte dos critérios para diagnóstico desse distúrbio, resultando em possíveis equívocos. Portanto, o tratamento deve ser individualizado e de caráter multifatorial, considerando as queixas da paciente, o estágio da vida reprodutiva em que se encontra e avaliando o risco/benefício de determinadas terapias.

\section{REFERÊNCIAS}

1. BATISTA LB, et al. Síndrome de ovários poliquísticos. Entidad que demanda diagnóstico temprano. Revista Médica Electrónica, 2019; 41(4): 1684-1824.

2. BLAY SL, et al. Polycystic ovary syndrome and mental disorders: a systematic review and exploratory meta-analysis. Neuropsychiatr Dis Treat. 2016; 12: 2895-2903.

3. BOZDAG G, et al. The prevalence and phenotypic features of polycystic ovary syndrome: a systematic review and meta-analysis. Human Reproduction, 2016; 31: 2841-2855.

4. DOKRAS A, et al. Weight Loss and Lowering Androgens Predict Improvements in Health-Related Quality of Life in Women With PCOS. The Journal of clinical endocrinology and metabolism, 2016; 101(8): 2966-74.

5. FREIRE A, et al. Síndrome de Ovario Poliquístico (SOP) en la adolescencia. Rev. Hosp. Niños, Buenos Aires, 2018; 258-263.

6. FUNG L, et al. Resistencia a la insulina en la mujer: ¿cómo y cuándo evaluarla? Rev Obstet Ginecol Venez, Caracas, 2015; 75(3): 200-211.

7. GOWRI BV, et al. Correlation of Skin Changes with Hormonal Changes in Polycystic Ovarian Syndrome: A Crosssectional Study Clinical Study. Indian J Dermatol., 2015; 60(4): 419.

8. HOEGER K, et al. The impact of metformin, oral contraceptives, and lifestyle modification on polycystic ovary syndrome in obsese adolescent women in two randomized, placebo-controlled clinical trials. Journal of Clinical Endocrinology Metabolic, 2008: 4299-4306.

9. IBÁÑEZA L, et al. Pathophysiology, Diagnosis, and Treatment of Polycystic Ovarian Syndrome in Adolescence. Horm Res Paediatr, 2017; 88(6): 371-395.

10. KOGURE GS, REIS RM. Progressive Resistance Training as Complementary Therapy for Polycystic Ovarian Syndrome. Revista Brasileira de Ginecologia e Obstetrícia, 2017; 39: 255-257.

11. LASS N, et al. Effect of lifestyle intervention on features of polycystic ovarian syndrome, metabolic syndrome and intima-media th in thickness obese adolescent girls. Journal of Clinical Endocrinology Metabolic, 2011; 96: 3533-3540.

12. McCARTNEY CR, MARSHALL JC. Clinical Practice: Polycystic ovary syndrome. The New England journal of medicine, 2016; 375(1): 54-64.

13. ORNSTEIN RM, COPPERMAN NM, JACOBSON MS. Effect of weight loss on menstrual function in adolescents with polycystic ovary syndrome. Journal of Pedriatic Adolescents Gynecology, 2011; 24: 161-165.

14. PEÑA GM, et al. Resistencia a la insulina en mujeres con síndrome de ovario poliquístico. Revista Cubana de Endocrinologia, 2019; 30(2): e179.

15. RODRÍGUEZ JH, PUIG MEL. Relación entre la insulinorresistencia y el síndrome de ovario poliquístico y sus comorbilidades metabólicas. Revista Cubana de Medicina General Integral, 2015; 31(4): 1561-3038.

16. ROSA-E-SILVA AC. Conceito, epidemiologia e fisiopatologia aplicada à prática clínica. In: Síndrome dos ovários policísticos. São Paulo: Federação Brasileira das Associações de Ginecologia e Obstetrícia (FEBRASGO), 2018 ; $1: 1$ 15.

17. TAY CT, et al. Updated adolescent diagnostic criteria for polycystic ovary syndrome: impact on prevalence and longitudinal body mass index trajectories from birth to adulthood. BMC Medicine, 2020; 18: 1-11.

18. VILLACIS SC, et al. Criterios diagnósticos y tratamiento integral terapéutico del síndorme de ovarios poliquísticos. Revista Cubana de Ginecologia y Obstetricia, 2017; 43: 173-181.

19. WINNYKAMIEN I, et al. Síndrome de ovário poliquístico. Revista del Hospital Italiano, Buenos Aires, 2017; 37(1): 1020.

20. WITCHEL SF, et al. The Diagnosis of Polycystic Ovary Syndrome during Adolescence. Hormone Research in Paedriatics, 2015; 83: 376-389.

21. YELA DA. Particularidades do diagnóstico e da terapêutica da síndrome dos ovários policísticos na adolescência. In: Síndrome dos ovários policísticos. São Paulo: Federação Brasileira das Associações de Ginecologia e Obstetrícia (FEBRASGO), 2018; 2: 16-28.

22. BLAY SL, et al. Polycystic ovary syndrome and mental disorders: a systematic review and exploratory meta-analysis. Neuropsychiatr Dis Treat. 2016; 12: 2895-2903. 